University of Wollongong

Research Online

Faculty of Engineering and Information

Faculty of Engineering and Information

Sciences - Papers: Part A

Sciences

$1-1-2016$

\title{
An intelligent driver alerting system for real-time range indicator embedded in electric vehicles
}

Kaveh Sarrafan

University of Wollongong, ks282@uowmail.edu.au

Kashem M. Muttaqi

University of Wollongong, kashem@uow.edu.au

Danny Sutanto

University of Wollongong, soetanto@uow.edu.au

Graham Town

Macquarie University

Follow this and additional works at: https://ro.uow.edu.au/eispapers

Part of the Engineering Commons, and the Science and Technology Studies Commons

Research Online is the open access institutional repository for the University of Wollongong. For further information contact the UOW Library: research-pubs@uow.edu.au 


\title{
An intelligent driver alerting system for real-time range indicator embedded in electric vehicles
}

\begin{abstract}
This paper proposes a state-of-the-art algorithm for a real time charging recommendation for an electric vehicle (EV) driver based on an accurate real-time range indicator system to avoid range anxiety. The charging recommendation algorithm alerts the driver when charging is deemed required for the selected route. This algorithm determines the nearest charging location obtained using GPS based on an accurate estimation of $\mathrm{SoC}$ at the destination and when charging determines the optimum charging time required by the battery to have sufficient energy to reach the destination. The graphical user interface (GUI) of the real-time range indicator system is also used to show the driver an accurate estimation of the remaining range to destination and the current state of charge $(\mathrm{SoC})$. The results from simulations of a range of routes validate the proposed algorithm.

\section{Disciplines}

Engineering | Science and Technology Studies

\section{Publication Details}

K. Sarrafan, K. M. Muttaqi, D. Sutanto \& G. Town, "An intelligent driver alerting system for real-time range indicator embedded in electric vehicles," in IEEE Industry Applications Society, 51st Annual Meeting: IAS 2016, 2016, pp. 1-7.
\end{abstract}




\section{An Intelligent Driver Alerting System for Real-time Range Indicator Embedded in Electric Vehicles}

\author{
Kaveh Sarrafan*, Kashem M. Muttaqi \\ and Danny Sutanto \\ School of Electrical, Computer and \\ Telecommunications Engineering \\ University of Wollongong, NSW, Australia \\ *ks282@uowmail.edu.au
}

$\begin{array}{ll}P_{L}(i) & \text { Load power (shaft power) at segment } \mathrm{i}(W) \\ P_{M}(i) & \text { Induction motor input power at segment } i(W) \\ P_{Q}(i) & \text { Total switch losses at segment } i(W) \\ P_{\text {rain }}(i) & \text { Rain probability at segment } i \\ P_{w}(i) & \text { Total traction power at segment } i(W) \\ \text { SoC }(\text { des }) & \text { SoC at the destination } \\ \text { So } C_{m} & \text { Minimum acceptable SoC for the electric vehicle } \\ \text { SoC }(i) & \text { SoC at segment } i \\ \text { So } C_{t} & \text { Threshold value above the minimum acceptable SoC } \\ t_{c} & \text { Recommended charging time }(\mathrm{min}) \\ v(i) & \text { Vehicle velocity at segment } i(\mathrm{~m} / \mathrm{sec}) \\ V_{B} & \text { Battery pack voltage }(\mathrm{V}) \\ v_{\text {rel }}(i) & \text { Relative vehicle speed at segment } i(\mathrm{~m} / \mathrm{sec}) \\ w_{v(i)} & \text { Wind speed at segment } i(\mathrm{~m} / \mathrm{sec}) \\ V_{a l u e_{c}} & \text { Necessary charging value at the segment } i \\ \alpha_{i} & \text { Slope angle at segment } i \\ \rho_{\text {air }} & \text { Density of air }\left(\mathrm{kg} . \mathrm{m}^{3}\right) \\ \eta_{I M}(i) & \text { Induction motor efficiency at segment } i \\ \eta_{I n v}(i) & \text { Inverter efficiency at segment } i\end{array}$

\section{INTRODUCTION}

From the EV buyer's perspective, the major problems with EVs are their limited battery capacity and the availability of charging infrastructure, which can cause range anxiety (driver's fear of driving an electric vehicle for a long distance) [1]. These concerns rank even higher than the higher cost of the vehicles and batteries. Even taking into consideration the new low-cost generation of electric vehicle batteries, the range anxiety can still limit the usage of electric vehicles. Recently, a great deal of effort has been devoted to the study of reducing range anxiety by improving the SoC/range estimation methods in the vehicle battery monitoring systems using low cost microcontrollers [2], taking environmental conditions into consideration [3] [4], driver behavior [3] [5] [6], traffic conditions [6]-[7] and, time-variant efficiency analysis of the drive system [8]-[9]. There is still a lack, however, of studies into the communication between the vehicle battery monitoring systems and the electric vehicle drivers.

Ferreira et al. [10] propose the SiREV recommender system for electric vehicle drivers. This system contains 
several modules, including a GPS module, an energy market information module, a public transportation information module, a vehicle to grid (V2G) system module, and a charging station reserve module. Based on the predefined selections of the user, the SiREV system will provide the information about charging mode, location type, nearby point of interest, etc. Based on the geographical position of the EV, the SiREV can find the nearest charging locations and can provide the distance to the charging stations. The authors concede, however, that, this model is only at the research stage and implementing it in real electric vehicles is extremely difficult and it will quite a challenge to establish an information exchange environment in a smart grid system and public transportation system.

Tannahill et al [3] studied the $\mathrm{SoC}$ estimation model along with the charging recommendation system. The proposed SoC estimation model in [3] considers all the environmental conditions and behavioral factors, but the authors concede that it is a preliminary paper and more research is needed into this innovative system. The recommendation algorithm has the ability to alert the driver when charging is required and can also provide an estimation of the duration of the charging required. The proposed charging recommendation system in this paper however, can alert the driver when charging is required during the route, identify the nearest charging stations with the exact names, addresses and geographical coordinates, and the amount and time required to charge the $\mathrm{EV}$ to reach the destination.

The remainder of the paper is organized into four sections. Section III presents the proposed real-time range indicator model with recommendation system. Section IV presents the details of the proposed GUI will be discussed. Section V summarizes the results of this work; and Section VI concludes the findings and identifies future works to be done.

\section{Proposed ReAL-time RANGe IndicAtor Model with RECOMMENDATION SYSTEM}

This paper proposes an improved model of a real-time range indicator system which takes into consideration all the environmental and behavioral factors. A smart data collection code is used to extract all the necessary data in real time using wireless internet connection from different sources in the internet, such as the Google Map, weather information bureau, etc. The GUI is then linked with the proposed realtime range indicator system to create a graphical view of the results along a selected route. Fig. 1 shows the real-time range indicator framework. Fig. 1 shows that an intelligent recommendation system is added to the range indicator system to alert the driver when the destination is beyond the estimated range of the electric vehicle (based on the initial charging condition and the driving pattern so far). The charging recommendation algorithm will then tell the driver which station is the best one to charge the battery in terms of distance based on the information of the available charging stations in the area obtained from GPS. The proposed charging recommendation system will also provide the charging time to reach the destination (including some margin), to reduce the time required to have the EV fully charged. Fig. 2 shows all the available charging stations currently available in Australia used in this paper. It is envisaged that as EV becomes more popular, more charging stations will be made available by local governments and private entities.

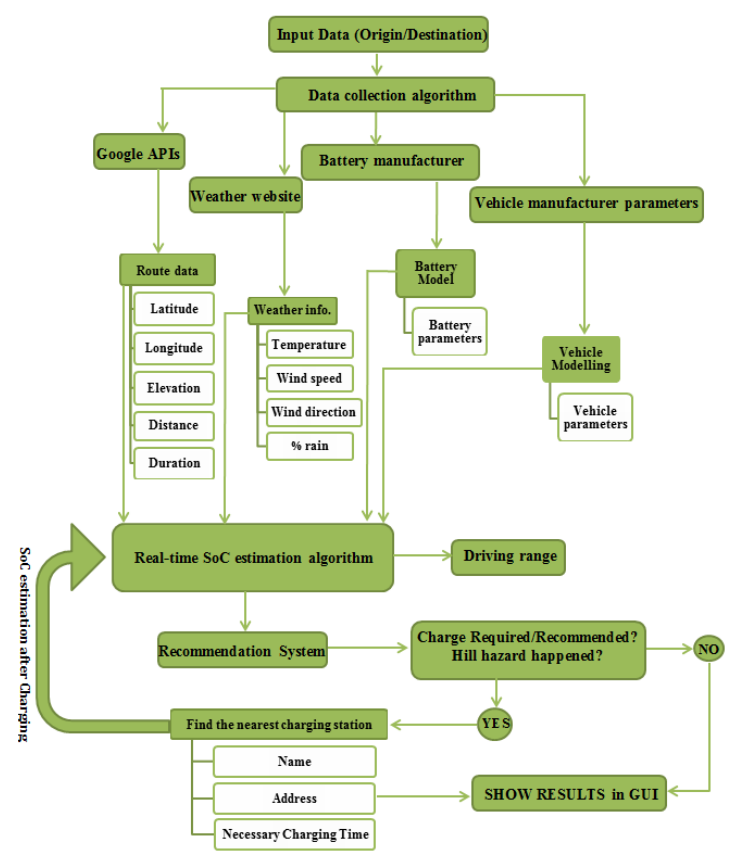

Fig. 1. The framework of the real-time range indicator system with charging recommendation system

\section{A. Data collection and battery power calculation}

To estimate the required $\mathrm{SoC}$ at the destination, it is essential to collect all the constant, historical and real-time data from different sources. Fig. 1 shows all the necessary input data such as the route data, the weather information, the battery model information, such as age and condition of the battery and vehicle parameters. Google APIs have been used to extract the route data and weather forecast websites have been used to extract the weather information. The data is then utilized for traction force calculation. In most real-time range indicator system, a large number of assumptions are usually made for the calculation of traction power [7] [12] [13]. For example, the route is assumed flat without any elevation profile or the effect of the wind speed and wind direction on the vehicle speed is ignored. To avoid errors due to these assumptions, the proposed method takes into consideration all environmental factors in real time for the calculation of traction force. For example, Fig. 3 shows how the external forces affect the electric vehicle while driving. 


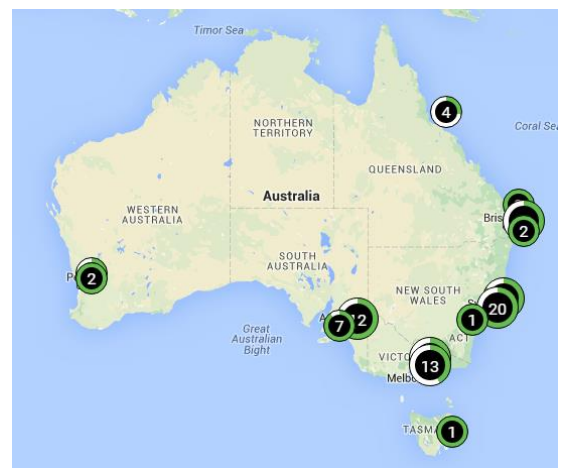

Fig. 2. Currently available charging stations in Australia [11]

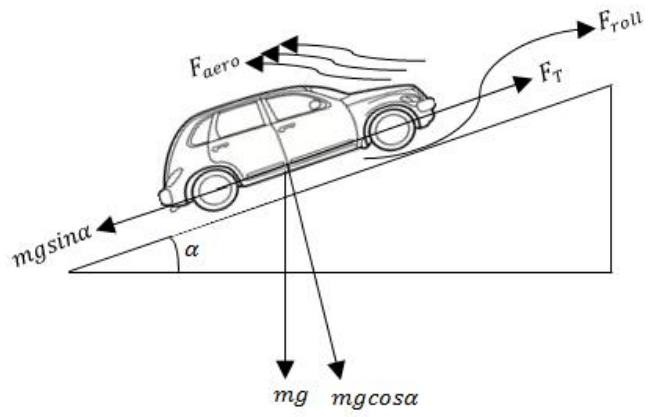

Fig. 3. Schematic view of the external forces acting on the electric vehicle during driving

In order to calculate the traction force along a route, a route segmentation is used. For each segment, the latitude and longitude of the start and end points, the wind speed and direction, the probability of rain, the elevation and the effective rolling resistance need to be considered. Fig. 4 demonstrates the power transition in an electric vehicle at segment $i$ along a route. Fig. 4 demonstrates that, in order to calculate the battery power along a route at each segment, it is essential to calculate traction power from the wheel side of the electric vehicle. Based on the transmission system gear ratio and the efficiency, the load power seen from the shaft of the induction motor can be calculated. In the proposed method the efficiency of the drive system (motor plus inverter) is calculated at any instant of time, unlike many papers where the authors consider the efficiency reported by the manufacturer data is assumed to be constant at any instant of time [3] [12]. In order to improve the SoC estimation, auxiliary electric loads such as that used for air conditioning, windscreen wipers and lighting are also taken into account. Fig. 4, shows how friction loss, gravity loss, aerodynamic loss and acceleration loss are taken into account during the calculation of the traction power/force [3] [12]. The full details of the traction power calculation along a segment of a route are given in Equations (1) - (3).

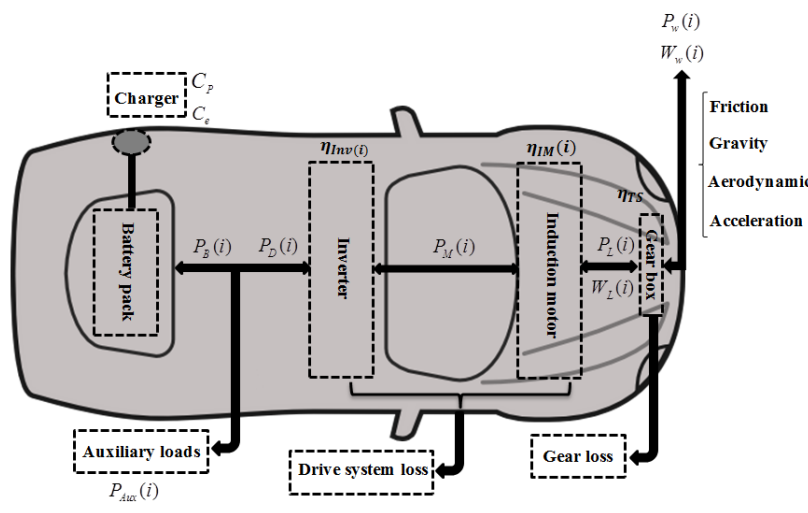

Fig. 4. Power transition in electric vehicle at segment $\mathrm{i}$ along a route

$$
\begin{aligned}
& F_{w}(i)=m\left(a(i)+g\left(\sin \alpha_{i}+C_{r r}(i) \cos \alpha_{i}\right)\right)+1 / 2 \rho_{\text {air }} C_{d} A v(i)_{r e l}^{2} \\
& v_{r e l}(i)=v(i)+\left(w_{v(i)} \times \cos \left(d i r_{E V(i)}-d i r_{w i n d}(i)\right)\right) \\
& C_{r e}(i)=C_{r d}+P_{r a i n}(i)\left(C_{r w}-C_{r d}\right)
\end{aligned}
$$

The traction power at segment $i$ along a route can be calculated using (4). The negative values for traction power calculation represent the power direction from the wheel side to the battery side of the electric vehicle (regenerative braking mode).

$$
P_{w}(i)=F_{W}(i) \cdot v(i)
$$

In this paper, the efficiency of the transmission system changes under different environmental conditions is assumed constant along a route due to the limited information available for the transmission system for the converted Hyundai Getz (blade electron) used in this paper.

$$
P_{L}(i)=\left\{\begin{array}{l}
P_{w}(i) / \eta_{T S}, P_{w}(i) \geq 0 \\
\eta_{T S} \times P_{w}(i), P_{w}(i)<0
\end{array}\right.
$$

The input inverter power in Fig. 4 can be calculated by evaluating the efficiency of the motor and drive system using (6).

$$
P_{D}(i)=\left\{\begin{array}{l}
P_{L}(i) /\left(\eta_{I M}(i) \times \eta_{I n v}(i)\right), P_{L}(i) \geq 0 \\
P_{L}(i) \times \eta_{I M}(i) \times \eta_{I n v}(i), P_{L}(i)<0
\end{array}\right.
$$

The efficiencies of the motor and inverter can be calculated using (7) and (8) respectively for both normal and regenerative braking conditions.

$$
\begin{aligned}
& \eta_{I M}(i)=\left\{\begin{array}{l}
P_{L}(i) /\left(P_{L}(i)+\left(P_{\text {Copper }}(i)+P_{\text {Core }}(i)\right)\right), P_{L}(i) \geq 0 \\
\left(P_{L}(i)-\left(P_{\text {Copper }}(i)+P_{\text {Core }}(i)\right)\right) / P_{L}(i), P_{L}(i)<0
\end{array}\right. \\
& \eta_{I n v}(i)=\left\{\begin{array}{l}
P_{M}(i) /\left(P_{M}(i)+\left(P_{Q}(i)+P_{D i}(i)\right)\right), P_{L}(i) \geq 0 \\
\left(P_{M}(i)-\left(P_{Q}(i)+P_{D i}(i)\right)\right) / P_{M}(i), P_{L}(i)<0
\end{array}\right.
\end{aligned}
$$

It should be noted that for each segment along a route, for the calculation of the induction motor losses, the motor would operate in the constant torque region or the constant power 
region, depending on the vehicle speed. Different mathematical calculations for each of these will be needed. The details of the loss calculation methods for the three-phase induction motor and inverter used in this paper are fully discussed in the authors' earlier work [9].

The necessary power from the battery side and the battery current for each segment along a route can be calculated as follows:

$$
\begin{aligned}
& P_{B}(i)=P_{A u x}(i)+P_{D}(i) \\
& I_{B}(i)=P_{B}(i) / V_{B}
\end{aligned}
$$

For the SoC estimation, the model proposed in [9] is used. Based on this model, by calculating the battery current from (10) and adding all the correction factors to estimate the corrected battery capacity, the expected state of charge along a route can be calculated. The model proposed in [9] has been verified with experimental tests and has been shown to agree with the experimental measurements.

\section{B. Charging recommendation algorithm}

To reduce the range anxiety, a driver alerting option needs to be included to the vehicle's range estimation system. The proposed recommendation system has the ability to alert the driver when a recharge is necessary during the route and provides the name and address of the nearest charging station. In the proposed algorithm, the effects of unexpected traffic conditions, sudden changes in vehicle speed due to driver behavior and the varying energy usage required to reach the nearest charging station, a sufficient margin (threshold) above the minimum acceptable SoC is considered. The details of the proposed recommendation algorithm are described in Fig. 5 .

As can be seen form Fig. 5, this algorithm checks the SoC value for each segment to be above the minimum value plus the margin. Three events may occur along the selected route and the driver may get one or more of the following three messages: "hill hazard", "stop required" or "stop recommended". The message "hill hazard" will appear along a route when, for each segment, the SoC value is smaller than both the minimum acceptable SoC for the electric vehicle, $\mathrm{SoC}_{m}$, and $\mathrm{SoC}$ at the destination, $\mathrm{SoC}(\mathrm{des})$, (considering the regenerative braking system). This situation will occur when the electric vehicle faces steep roads with significant change in elevation. In this case, the charging status may go below the acceptable minimum range. When travelling downhill, instead, because of the regenerative braking system, the electric vehicle will be charged during braking. In this situation, the driver will be alerted at the starting point to charge the battery in order to prevent damage to the battery. The "stop recommended" message will happen along a route when $\mathrm{SoC}$ at the destination, $\operatorname{SoC}($ des), is smaller than the minimum acceptable $\mathrm{SoC}$ for the electric vehicle, $S o C_{m}$, plus the margin (threshold) value, SoC $C_{t}$. Finally, the "stop required" message will occur along a route when SoC at the destination, SoC(des), is smaller than the minimum acceptable SoC for the electric vehicle, $S_{o} C_{m}$, as shown in Fig. 5.

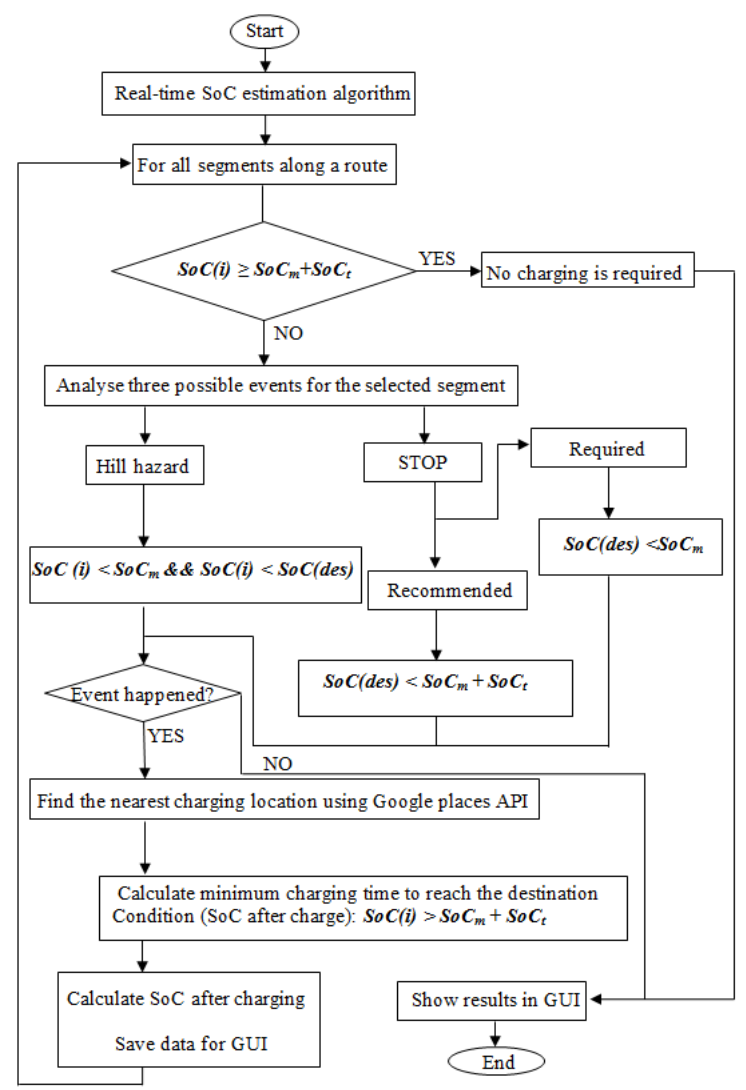

Fig. 5. The flowchart of the proposed charging recommendation algorithm

Based on these three events, the driver needs to make a decision to recharge the battery. This means that the charging recommendation system will now tell the driver the nearest charging station and its name and address using the Google places API. This algorithm will also tell the driver roughly how much time is necessary to charge the battery in order to reach the destination. If the selected route is beyond the ability of the electric vehicle or if the initial charge of the electric vehicle is at critical level, the charging algorithm will inform the driver to fully charge the battery. At this stage, the algorithm will calculate the SoC after charging and check the values again. If the three events occur again along the rest of the journey, it means that the vehicle needs to recharge the battery again and therefore the algorithm will restore the second charging station information to alert the driver. The charging time along a route can be calculated using (11) to (13). If the vehicle needs a full charge to reach the destination, (13) will be used. Otherwise, only the minimum possible charging time will be calculated to reach the destination using (12). 


$$
t_{C}=\left(\text { Value }_{C} \times B C \times 60\right) /\left(C_{P} \times C_{e}\right)
$$

For normal condition: (minimum charging time)

$$
\begin{aligned}
& \text { Value }_{c}=S o C_{m}+\operatorname{SoC}_{t}-\operatorname{Min}(\operatorname{SoC}) \\
& \text { For full-charge condition: }
\end{aligned}
$$

$$
\text { Value }_{c}=100-\operatorname{SoC}(i)
$$

\section{GRAPHICAL USER INTERFACE (GUI)}

To implement the proposed range indicator system with the intelligent charging recommendation algorithm in a real electric vehicle, it would be beneficial to have a graphical view of the proposed model in the simulation environment. In order to do this, the proposed algorithm code is linked with a proposed GUI device. In the proposed GUI device, the driver enters the origin and destination names of the selected route from which the GPS will determine the geographical positions (latitude, longitude) of the beginning and ending of the route, the initial SoC, the number of occupants and also the time of day in the corresponding boxes in the GUI. The user also has the option of avoiding tolls along a route. By pressing the "Get Route Data" button, the range indicator algorithm will extract all necessary real-time data from various sources. The Google direction API, Google elevation API, Google matrix distance API, WUI API, and Google places API are used for the real-time data extraction.

The designed GUI has the capability to show the road map of the selected origin and destination points. It can also show the accurate range to destination, and accurate $\mathrm{SoC}$ of the vehicles taken into account all the environmental conditions, based on the initial charging level and also the selected route. If the electric vehicle needs to be charged (because the SoC has fallen below the specified threshold), the algorithm will find the nearest charging station name, address and an accurate view of the corresponding charging station position obtained from the Google Map. From the pop-up menu in the "Route Results" panel, the users can select the simulated SoC (with/without charging), the elevation profile of the selected route, the estimated rate of change of the SoC, the estimated system efficiency and the available range along the selected route. Other parameters such as the ambient temperature, the estimated final value for the $\mathrm{SoC}$ with/without charging, the wind speed, the total distance, the probability of rain and the estimated duration time to reach the destination without charging. The alerting system will also notify the driver to charge the battery with the minimum necessary charging time to reach the destination if at least one of the three events mentioned in Section III occurs.

\section{REsults OF THE PRoposed REAL-TIME CHARgING RECOMMENDATION ALGORITHM}

To validate the proposed algorithm, the route between 6 Tigway Avenue, Figtree, NSW and 2 Compton Green, West
Pennant Hills, NSW in Australia was used. The parameters of the blade electron (Hyundai Getz) electric vehicle are used in this simulation for traction power estimation [9]. In this paper, the verified SoC estimation model proposed in [9] considering different environmental conditions along with time-domain efficiency analysis of the drive system were used. Only two occupants with the initial SoC of $94.7 \%$ was considered for this estimation. By pressing the "Get Route Data" button, the simulated GUI device will compile all the input data to run a real-time range indicator system. Meanwhile, the range indicator system will extract all the necessary real-time data using various APIs. Based on the time of the day, the number of occupants in the electric vehicle and the initial charging status, the range/SoC estimator will calculate the necessary range/SoC at the destination. If one of the three events mentioned in Section III happen along a route, the charging recommendation system will find the name, address and the position of the nearest charging station. In addition, the proposed algorithm will calculate the minimum charging time for the selected route to have an acceptable charging level at the destination (above the minimum acceptable value plus the threshold value). In this simulation, in order to increase the possibility of finding charging station points near to the vehicle position along a route, some petrol stations in Australia have been added to the charging stations presented in Fig. 2. Fig. 6 demonstrates the results of the selected route in GUI. As can be seen from Fig. 6, the range estimation algorithm selects the shortest route between the origin and the destination (also if the avoid tolls check box is selected, the algorithm will select another fastest route without tolls). The total distance and total time for the selected route were 127.9 kilometer and 87.98 minutes respectively (the traffic conditions were not considered in this paper). The average ambient temperature was 23 degree centigrade, the probability of the rain was 47 $\%$ and the wind speed was roughly $7.15 \mathrm{~m} / \mathrm{s}$. The results of the "Route Results" panel in GUI are shown in Fig. 7.

By scrutinizing the results in Fig. 6, it can be seen that for the selected route, the initial charging level at the origin point is not enough to reach the destination. Hence, the proposed charging recommendation system will alert the driver that charging is "required" along the route.

This algorithm selects the nearest charging location (when it is necessary) with the charging time along a route. At the bottom left of the Fig. 6, the total route is shown in road-map view. The "Alerting System" panel shows that there will be a hill hazard along the route with this charging condition. The $\mathrm{SoC}$ values at the destination with and without charging are $25.54 \%$ and $-42.28 \%$ respectively. If the driver recharges the battery of the electric vehicle at the recommended places for the suggested duration time, the final $\mathrm{SoC}$ at the destination will be above the appropriate predefined range. For this selected route, only one charging station is recommended (see Fig. 8). 

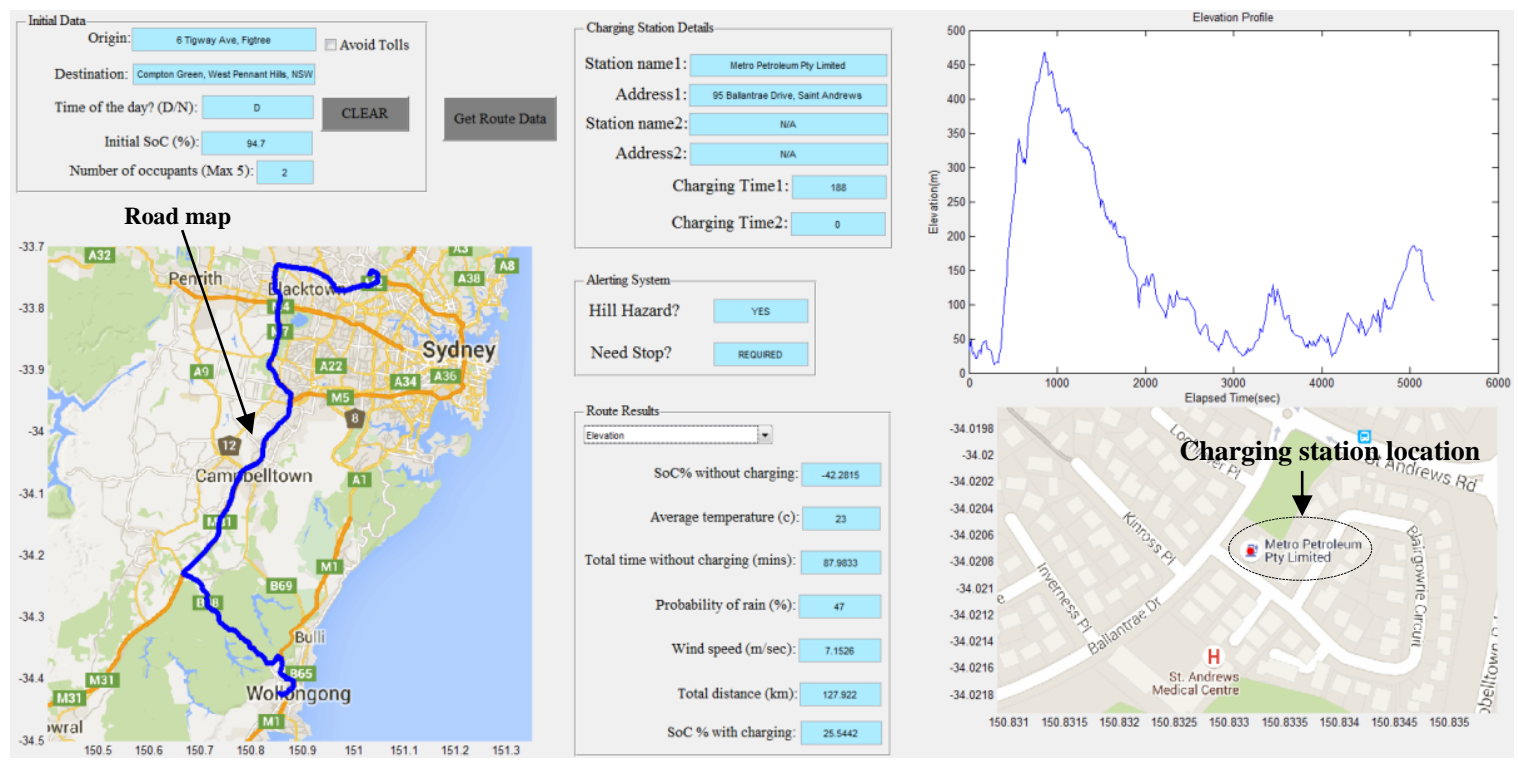

Fig. 6. The results of the proposed range indicator system for the selected route in GUI

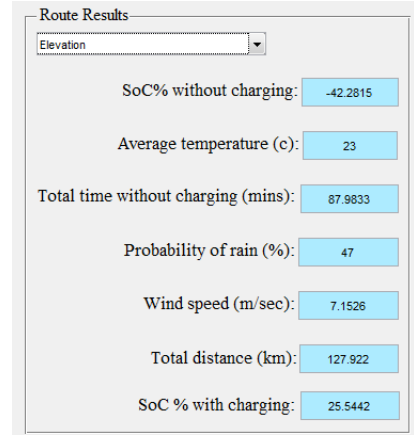

Fig. 7. Route results panel

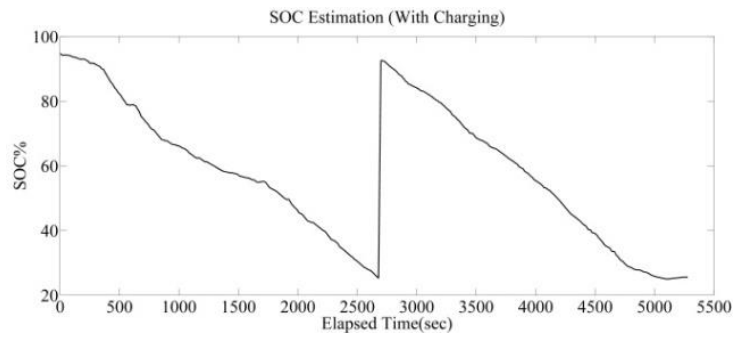

(a)

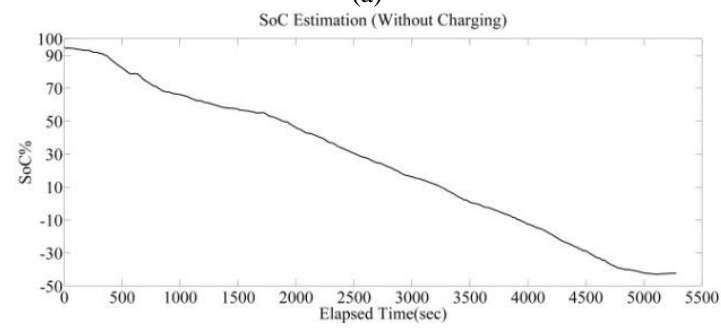

(b)

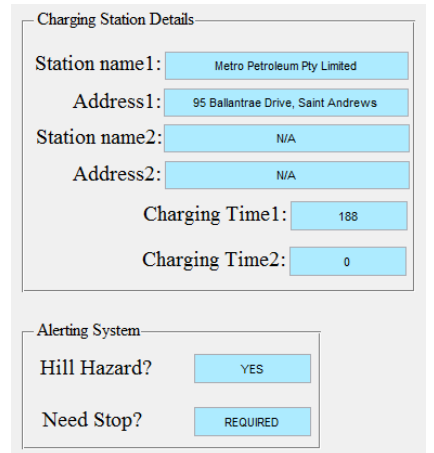

Fig. 8. Charging station details and alerting system panels

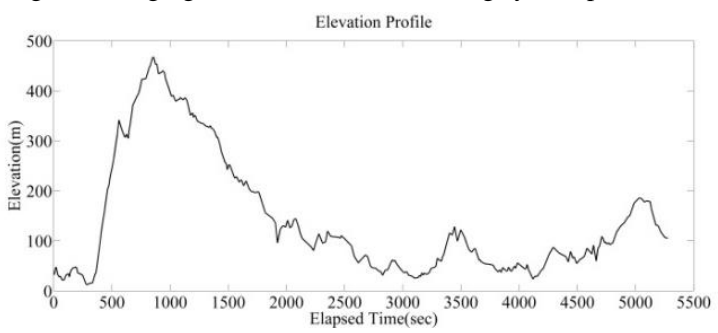

(c)



(d)

Fig. 9. The plotted results of the popup menu in "Route Results" panel for the selected route

(a) SoC estimation with charging (b) SoC estimation without charging (c) The elevation profile (d) time-variant drive system efficiency analysis 
At the first charging location, the electric vehicle battery needs a full charge for about 188 minutes. At the bottom right of Fig. 6, a charging location along with its latitude and longitude information is presented (red point). The driver can also "zoom in" to the map to have a closer view with more details of the charging station positions. The plotted results of the pop-up menu in the "Route Results" panel are demonstrated in Fig. 9.

Fig. 9(a) and Fig. 9(b) show the SoC estimation with and without charging, respectively. As can be seen from the Fig. 9(a), between 2000 and 3000 seconds, the battery of the EV is fully charged. The instant charging in Fig. 9(a) reveals that in this algorithm the charging time is considered separately, as was explained in Section III and charging time in Charging Station Details tab in Fig. 6 needs to be added to the elapsed time. Fig. 9(c) shows the elevation profile of the selected route varies between $12 \mathrm{~m}$ and $467 \mathrm{~m}$. Fig. 9(d) reveals that the system efficiency is not constant along the route and, in some cases; the system efficiency is zero because, in these cases, the electric vehicle is in regenerative braking mode, but the traction power is smaller than the constant losses in the threephase induction motor. For this reason, all the braking power is used for reducing the vehicle speed or stopping it.

\section{CONCLUSION AND FUTURE WORK}

A real-time range/SoC estimation model and an intelligent recharge alerting system are proposed in this paper to reduce the driver range anxiety. An intelligent algorithm and a proposed GUI device are used in this model to alert the driver the need to recharge once the $\mathrm{SoC}$ level drops below a certain threshold. If one of the three events (hill hazard, stop required and stop recommended) occurs along a route, the charging recommendation algorithm has the ability to show the two nearest charging locations with their names, addresses and GPS coordinates. This algorithm also calculates the minimum charging time required at the charging station to reach the destination. The proposed system will enhance the use of electric vehicles by reducing range anxiety and reduce the necessary charging time along a route. The proposed algorithm also helps the driver to travel over a longer distance without the possibility of being stranded by a depleted electric battery. Further research work will need to include the development of an actual real-time range indicator system with an actual GUI device (rather than the simulated ones) that can also consider traffic congestion and driver behavior.

\section{ACKNOWLEDGMENT}

The authors acknowledge the Australian Power Quality and Reliability Center for providing research facilities. This research is supported by the Australian Research Council (ARC) and Optus through the ARC Linkage Project Scheme, LP130100811.

\section{REFERENCES}

[1] E. Ona, and S. Long, "Barriers to widespread adoption of electric vehicles: An analysis of consumer attitudes and perceptions," Energy policy, vol. 48, pp. 717-729, 2012.

[2] V. R. Tannahill, D. Sutanto, K. M. Muttaqi, and M. A. Masrur, "Future vision for reduction of range anxiety by using an improved state of charge estimation algorithm for electric vehicle batteries implemented with low-cost microcontrollers," IET Electr. Syst. Transp., vol. 5, pp. 24-32, 2015.

[3] V. R. Tannahill, K. M. Muttaqi, and D. Sutanto, "Driver alerting system using range estimation of electric vehicles in real time under dynamically varying environmental conditions," IET Electr. Syst. Transp., pp. 1-10, 2015.

[4] P. Roman, and J. P. Lebacque, "Breadth-First Search-Based Remaining Range Estimation and Representation for Electric Vehicle," SAE Technical Paper, No. 2014-01-0273, 2014.

[5] E. Kim, J. Lee, and K. Shin, "Real-Time Prediction of Battery Power Requirements for Electric Vehicles," in Proc. 2013 Cyber-Physical Systems Conf., pp. 11-20.

[6] R. Wang and S. Lukic , "Review of driving conditions prediction and driving style recognition based control algorithms for hybrid electric vehicles", in Proc. 2011 IEEE VPPC, pp.1 -7.

[7] R. Shankar, and J. Marco, "Method for estimating the energy consumption of electric vehicles and plug-in hybrid electric vehicles under real-world driving conditions," IET Intel. Transport Syst., 7 (1), pp. 138-150, 2013.

[8] S. S. Williamson, A. Emadi, and K. Rajashekara, "Comprehensive Efficiency Modeling of Electric Traction Motor Drives for Hybrid Electric Vehicle Propulsion Applications," IEEE Trans. Vehicular Technology, vol. 56, pp. 1561-1572, 2007.

[9] K. Sarrafan, D. Sutanto, K. M. Muttaqi, and G. Town, "Accurate range estimation for an electric vehicle including changing environmental conditions and traction system efficiency," IET Electr. Syst. Transp., May 2016.

[10] F. João, P. Pereira, P. Filipe, and J. Afonso, "Recommender system for drivers of electric vehicles." In Proc. 2011 IEEE Electronics Computer Technology (ICECT) Conf., pp. 244-248.

[11] '-chargepoint +',https://www.chargepoint.net.au/charge_point,Accessed April 2016.

[12] W. Vaz, A.K.R. Nandi, R.G. Landers, and U. O. Koylu, "Electric vehicle range prediction for constant speed trip using multi-objective optimization," J. Power Sources, 275, pp. 435-446, 2015.

[13] R. Prins, R. Hurlbrink, and L. Winslow, "Electric vehicle energy usage modelling and measurement," IJME, 13 (1), pp. 5-12, 2013. 